\title{
Design and Implementation of Intelligent Tutoring System using Enhanced Personalization in e-Learning
}

\author{
Smruti Nanavaty
}

\begin{abstract}
A review of different E-learning Experience considers the need for personalization of learning contents to enhance the experience of learners. The current scenario of education shows that more and more learners are using e-learning to earn their degrees, build upon their knowledge base and acquire new skills. E-Learning is a $\$ 56.2$ billion industry today and will double by 2017. Statistics show that by 2019, roughly half of all educational institutions will offer e-Learning based training. This initiates the study of various methodologies which analyze the profiles, learning styles, behavior, and capabilities for mapping the appropriate learning content to appropriate user. A review of approaches and methods was conducted by studying articles of past9 years (2006-2015) by extracting information for techniques used for improving e-learning experience. A five stage literature review of personalization of learning contents using various approaches was conducted. The strengths and weaknesses followed by gaps in the related work are discussed. Further an intelligent tutoring model is proposed as the solution to enhance personalisation in e-learning.
\end{abstract}

Keywords: e-Learning, personalization, data mining, learning styles, contents and behaviour, e-learning platforms, intelligent tutoring systems, recommender system, blended approach

\section{Introduction}

The present research is presented with a view to study personalization and adaptation of learning contents to the elearners based on their requirements. With the advance in IT, human knowledge and learning content have an incredible increase in the quantity and variety of digital content. The trends have implications on the quality and relevance of knowledge and learning content delivered to organization workers and e-learners. The benefits of using elearning are obvious but the process is effective only if the learner is provided with appropriate learning objects, aligned with his learning style, capabilities and requirements. As a result of this growing online knowledge and learning content there is an urgent need of designing learner centric elearning systems.

There are many factors that influence the extent of learning. These would include factors such as learner"es learning style and motivation for learning. An important role of e-learning content providers is to recognize that their pedagogy and educational material must cater for the individual learnere $s$ requirements. There is an immediate need to move away from "one size that fits all" paradigm and offer personalized learning experience. Based on reviews undertaken for improving the e-learning experience, a comprehensive approach for enhancing the e-learning experience is proposed.

\section{Literature Review}

Instructors use various tools to deliver the online contents to e-learners. The challenge for content developers is to provide appropriate content to the users to satisfy their individual needs.

Improving e-Learning experience through Personalization of e-learning contents

This method deals with providing appropriate contents to the learners after analyzing the learneres needs and capabilities. Static Personalization deals with collecting the necessary data from the user and then analyzing the data using techniques of Data Mining to find individual needs and providing learning contents useful to them. Dynamic Personalization involves studying and analyzing the behavior and capabilities of the users and then dynamically mapping the contents to the user. Intelligent Tutoring Systems and Recommender System considerdeveloping a middleware or an agent based model to use the data from the learning systems to provide a recommendation for the requirements of the learners.Major challenge for e-content designers is that the content should be user specific and should satisfy the needs of various different learners. Moreover it is difficult to dynamically map the contents to the user"s specific needs and the users may not be able to specify the needs correctly.

\section{Key Findings with Solution Approaches}

This section discusses solution approaches which have been used by the researchers to validate or simulate their results and findings, the type of methodologies adopted, technology platform and details of hardware/ software used to obtain or validate their results 


\section{International Journal of Science and Research (IJSR) \\ ISSN (Online): 2319-7064}

Index Copernicus Value (2015): 78.96 | Impact Factor (2015): 6.391

Table 6: Personalization using Intelligent Tutoring and Recommender Systems

\begin{tabular}{|c|c|c|c|c|c|c|c|c|c|}
\hline $\begin{array}{l}\text { Ref. No. } \\
\text { Study type }\end{array}$ & Purpose & $\begin{array}{l}\text { Data } \\
\text { Input }\end{array}$ & Data source & Data size & $\begin{array}{c}\text { Parameters } \\
\text { studied }\end{array}$ & Methodology & $\begin{array}{c}\text { Software } \\
\text { /Tools }\end{array}$ & $\begin{array}{c}\text { Performance } \\
\text { Parameters }\end{array}$ & Results \\
\hline $\begin{array}{c}{[15]} \\
\text { Prototype } \\
\text { Design and } \\
\text { evaluation }\end{array}$ & $\begin{array}{c}\text { Multi-agent } \\
\text { system for } \\
\text { Web } \\
\text { Intelligent } \\
\text { Tutoring }\end{array}$ & $\begin{array}{c}\text { Student } \\
\text { Profile } \\
\text { Student } \\
\text { feedback }\end{array}$ & $\begin{array}{c}\text { User Profile } \\
\text { database } \\
\text { Questionnaire } \\
\text { from students } \\
\text { of Southwest } \\
\text { University of } \\
\text { China } \\
\end{array}$ & 320 & $\begin{array}{c}\text { Frequency of } \\
\text { use } \\
\text { Interactivity } \\
\text { Efficiency } \\
\text { Usefulness } \\
\text { Convenience }\end{array}$ & $\begin{array}{l}\text { Mathematical } \\
\text { Model for } \\
\text { recommendation } \\
\text { Statistical } \\
\text { methods for } \\
\text { evaluation }\end{array}$ & $\begin{array}{l}\text { Mathematical } \\
\text { equation }\end{array}$ & $\begin{array}{c}\text { Strongly } \\
\text { disagree to } \\
\text { strongly } \\
\text { agree: } 5 \text { point } \\
\text { scale }\end{array}$ & \begin{tabular}{|c|} 
Average \\
for \\
Personalize \\
d learning : \\
4.43
\end{tabular} \\
\hline $\begin{array}{c}{[4]} \\
\text { Comparativ } \\
\text { e study of } \\
\text { AEH }\end{array}$ & \begin{tabular}{|c|} 
Quantitative \\
approach for \\
Evaluating \\
Learning \\
Style AEH
\end{tabular} & \begin{tabular}{|c|} 
AEH \\
Systems \\
features
\end{tabular} & \begin{tabular}{|l|} 
Random \\
selection
\end{tabular} & $\begin{array}{l}24 \mathrm{AEH} \\
\text { Systems }\end{array}$ & $\begin{array}{l}\text { Intervention } \\
\text { time } \\
\text { Minimum } \\
\text { group size } \\
\text { Scientific } \\
\text { objectivity }\end{array}$ & 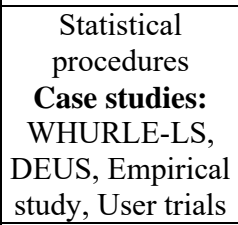 & \begin{tabular}{|c|} 
Correlation \\
using multiple \\
regression, \\
SPSS, t-test, \\
Chi-Square, \\
ANOVA \\
\end{tabular} & $\begin{array}{l}\text { Approach: } \\
\text { Equal } \\
\text { Greater } \\
\text { Important }\end{array}$ & $\begin{array}{c}\text { Compariso } \\
\text { n Table }\end{array}$ \\
\hline $\begin{array}{l}{[14]} \\
\text { Experiment } \\
\text { al study }\end{array}$ & $\begin{array}{l}\text { Ontology } \\
\text { Extraction } \\
\text { Method for } \\
\text { Adaptive } \\
\text { Learning }\end{array}$ & $\begin{array}{c}\text { Short } \\
\text { messages } \\
\text { on online } \\
\text { discussio } \\
\mathrm{n} \text { forum } \\
\text { for } 10 \\
\text { minutes } \\
\text { Question } \\
\text { naire for } \\
\text { assessme } \\
\mathrm{nt}\end{array}$ & $\begin{array}{l}\text { Postgraduate } \\
\text { students of } \\
\text { management } \\
\text { course }\end{array}$ & \begin{tabular}{|c|} 
Topics 1 \\
to 10 and \\
topics 41 \\
to 50 First \\
$1000 \mathrm{web}$ \\
pages \\
retrieved \\
via \\
Google \\
Search \\
API
\end{tabular} & $\begin{array}{c}\text { Accuracy } \\
\text { Cohesiveness } \\
\text { Isolation } \\
\text { Hierarchy } \\
\text { Readability }\end{array}$ & \begin{tabular}{|} 
Context sensitive \\
text mining \\
Fuzzy domain \\
ontology \\
extraction \\
algorithm \\
Concept \\
extraction : BMI \\
method \\
Relation \\
extraction : SSIM \\
method
\end{tabular} & $\begin{array}{c}\text { Java Server } \\
\text { Pages } 2.1 \\
\text { Servlet } 2.5 \\
\text { TouchGraph } \\
\text { Apache } \\
\text { Tomcat } 6.0 \\
\text { web server }\end{array}$ & $\begin{array}{c}\text { Mean score } \\
\text { For concept } \\
\text { map } \\
\text { Assessment: } \\
\text { Very Good } \\
\text { Good } \\
\text { Average } \\
\text { Bad } \\
\text { Very poor }\end{array}$ & \begin{tabular}{|c|} 
Mean \\
scores : \\
Accuracy : \\
4.23 \\
Cohesivene \\
ss: 4.22 \\
Isolation : \\
4.15 \\
Hierarchy : \\
4.31 \\
Readability \\
$: 3.95$ \\
\end{tabular} \\
\hline $\begin{array}{c}{[17]} \\
\text { Experiment } \\
\text { al }\end{array}$ & $\begin{array}{l}\text { Motivation } \\
\text { Prediction }\end{array}$ & $\begin{array}{c}\text { - Intrinsic } \\
\text { - extrinsic } \\
\text { motivatio } \\
\text { n data }\end{array}$ & $\begin{array}{l}\text { Questionnaire } \\
\text { data of } \\
\text { students for } \\
\text { behavior } \\
\text { pattern }\end{array}$ & 180 & $\begin{array}{l}\text { Motivation } \\
\text { Indexes: } \\
\text { - Autonomous } \\
\text { - Controlled } \\
\text { - e-learning } \\
\text { motivation, } \\
\text { - no. of hits }\end{array}$ & $\begin{array}{c}\text { - Likert type scale } \\
\text { - P- value } \\
\text { - statistical } \\
\text { procedures } \\
\text { - Correlation for } \\
\text { motivation Index }\end{array}$ & SPSS & $\begin{array}{c}\text { - Fairly } \\
\text { constant } \\
\text { - Slightly } \\
\text { irregular } \\
\text { - Quite } \\
\text { irregular }\end{array}$ & \begin{tabular}{|c|} 
Positive \\
correlation \\
of extrinsic \\
factor for \\
controlled \\
Motivation
\end{tabular} \\
\hline $\begin{array}{l}{[24]} \\
\text { Experiment } \\
\text { al study }\end{array}$ & \begin{tabular}{|c|} 
Mining \\
educational \\
data to \\
improve \\
adaptation \\
in e-learning
\end{tabular} & $\begin{array}{c}\text { Normalis } \\
\text { ed } \\
\text { Learners } \\
\text { log, } \\
\text { resources } \\
\text { info., } \\
\text { activities } \\
\text { log, }\end{array}$ & $\begin{array}{c}\text { LMS } \\
\text { MOODLE }\end{array}$ & $\begin{array}{c}66 \\
\text { students }\end{array}$ & $\begin{array}{c}\text { Effect of } \\
\text { Algorithmic } \\
\text { Induction of } \\
\text { Decision } \\
\text { trees, pruning } \\
\text { tactics on } \\
\text { classification } \\
\text { accuracy }\end{array}$ & $\begin{array}{l}\text { Data Clustering: } \\
\text { KSimpleMeans } \\
\text { Clustering } \\
\text { Data } \\
\text { Classification : } \\
\text { ID3-Decision } \\
\text { Tree }\end{array}$ & $\begin{array}{l}\text { J48 Algorithm } \\
\text { Clustering, } \\
\text { Multivariate } \\
\text { Analysis }\end{array}$ & $\begin{array}{c}\text { Learning style } \\
\text { wise } \\
\text { clustering } \\
\\
\text { Ranked } \\
\text { attributes }\end{array}$ & \begin{tabular}{|c|} 
Clustered \\
Instances \\
Concrete \\
LS: $38 \%$ \\
Concept \\
LS; $35 \%$ \\
Observe \\
LS : $21 \%$ \\
Experiment \\
$: 6 \%$ \\
Highest \\
Rank \\
0.72531
\end{tabular} \\
\hline $\begin{array}{c}{[5]} \\
\text { Experiment } \\
\text { al study }\end{array}$ & \begin{tabular}{|c|} 
Personalised \\
e-learning \\
LearnFit \\
using \\
dynamic \\
learner's \\
personality \\
\end{tabular} & \begin{tabular}{|c|} 
Students \\
learning \\
style and \\
preferenc \\
e using a \\
set of 60 \\
questions \\
\end{tabular} & $\begin{array}{c}\text { Students of } \\
\text { Computer } \\
\text { Information } \\
\text { Systems at } \\
\text { FSSM, } \\
\text { UCAM, } \\
\text { Morocco }\end{array}$ & \begin{tabular}{|c|} 
Control \\
Group : 24 \\
Experime \\
ntal Group \\
$: 24$
\end{tabular} & $\begin{array}{l}\text { Post test : } \\
\text { Mean Score } \\
\text { Standard } \\
\text { deviation } \\
\text { T value } \\
\text { P value }\end{array}$ & $\begin{array}{l}\text { Student t-test, } \\
\text { Kolmogrov- } \\
\text { Smirnov-test for } \\
\text { checking } \\
\text { distributions }\end{array}$ & $\begin{array}{c}\text { Mathematical } \\
\text { model }\end{array}$ & Post test score & $\begin{array}{c}\mathrm{T} \text { value }= \\
-4.53 \\
\mathrm{P} \text { value }= \\
0.02\end{array}$ \\
\hline $\begin{array}{l}\qquad 7] \\
\text { Experiment } \\
\text { al Analysis }\end{array}$ & \begin{tabular}{|c|} 
Student \\
Learning \\
profile \\
identificatio \\
$\mathrm{n}$ based on \\
user context \\
of \\
interaction
\end{tabular} & \begin{tabular}{|c|} 
Forum \\
logs \\
Discussio \\
ns logs \\
Exercise \\
logs \\
Question \\
naire logs
\end{tabular} & $\begin{array}{l}\text { LMS logs of } \\
\text { civil, } \\
\text { computer and } \\
\text { electric } \\
\text { engineering } \\
\text { students of } \\
2010 \text { batch }\end{array}$ & 297 & $\begin{array}{l}\text { Perceptive } \\
\text { Formative } \\
\text { Participative } \\
\text { styles based } \\
\text { on FSMLS }\end{array}$ & $\begin{array}{c}\text { Distributions } \\
\text { tabulated using } \\
\text { mathematical } \\
\text { algorithm } \\
\text { Least Square } \\
\text { Approximation } \\
\text { Student brain } \\
\text { model } \\
\text { K Nearest } \\
\text { Neighbor for } \\
\text { classification }\end{array}$ & $\begin{array}{c}\text { Mathematical } \\
\text { model }\end{array}$ & $\begin{array}{c}\text { No. Of } \\
\text { learning style } \\
\text { profiles : } \\
\text { Intuitive } \\
\text { Sensory } \\
\text { Verbal } \\
\text { Visual } \\
\text { Reflexive } \\
\text { Active }\end{array}$ & \begin{tabular}{|c|} 
Perceptive \\
Intuitive : \\
37 \\
Sensory: \\
260 \\
Formative \\
Verbal : 51 \\
Visual : \\
246 \\
Participati \\
ve \\
Reflexive : \\
7 \\
Active : \\
290
\end{tabular} \\
\hline
\end{tabular}




\section{International Journal of Science and Research (IJSR) \\ ISSN (Online): 2319-7064}

Index Copernicus Value (2015): 78.96 | Impact Factor (2015): 6.391

\begin{tabular}{|c|c|c|c|c|c|c|c|c|c|}
\hline $\begin{array}{c}{[27]} \\
\text { Case study }\end{array}$ & $\begin{array}{c}\text { Disengagem } \\
\text { ent } \\
\text { prediction }\end{array}$ & \begin{tabular}{|} 
Problem \\
solving \\
activity \\
data, test \\
and quiz \\
data
\end{tabular} & \begin{tabular}{|c} 
Study 1: \\
Log file from \\
HTML-Tutor \\
Study 2: \\
iHelp data \\
university of \\
Saskatchewan \\
\\
\end{tabular} & \begin{tabular}{|l} 
Study 1: \\
11 \\
students, \\
108 \\
sessions, \\
450 \\
sequences \\
Study 2: \\
21 \\
students, \\
218 \\
sessions, \\
735 \\
sequences \\
\end{tabular} & $\begin{array}{l}\text { Accuracy, } \\
\text { True positive } \\
\text { rates }\end{array}$ & $\begin{array}{l}\text { Simple Logistic } \\
\text { Classification } \\
2 \text { validation } \\
\text { studies } \\
\text { Statistical } \\
\text { methods } \\
\text { Pair t-test }\end{array}$ & $\begin{array}{c}\text { WEKA } \\
\text { Chi-Square } \\
\text { Evaluator }\end{array}$ & $\begin{array}{c}\text { Mean } \\
\text { Significant } \\
\text { Difference }\end{array}$ & $\mathrm{MSD}=3.0$ \\
\hline $\begin{array}{c}{[6]} \\
\text { Experiment } \\
\text { al study }\end{array}$ & \begin{tabular}{|c|} 
Document \\
Recommend \\
ation Model
\end{tabular} & $\begin{array}{c}\text { Data set: } \\
\text { Documen } \\
\text { ts }\end{array}$ & \begin{tabular}{|c|} 
Social \\
networks and \\
E-commerce \\
site
\end{tabular} & $\begin{array}{c}\text { Topics }= \\
50 \\
\text { Test } \\
\text { dataset }= \\
100\end{array}$ & $\begin{array}{c}\text { Predictive } \\
\text { utility based } \\
\text { on Document } \\
\text { Ranks }\end{array}$ & \begin{tabular}{|c|} 
Latent Dirichlet \\
Allocation for \\
Document-Topic \\
Coefficient \\
Matrix \\
Integrated \\
Recommendation \\
Algorithm built
\end{tabular} & - & $\begin{array}{l}\text { Similarity } \\
\text { between } \\
\text { documents } \\
\text { viewed \& } \\
\text { unseen } \\
\text { documents }\end{array}$ & $\begin{array}{c}\text { Highest } \\
\text { Predictive } \\
\text { Utility }\end{array}$ \\
\hline $\begin{array}{c}{[23]} \\
\text { Experiment } \\
\text { al study }\end{array}$ & $\begin{array}{c}\text { User centric } \\
\text { retrieval of } \\
\text { Learning } \\
\text { objects }\end{array}$ & \begin{tabular}{|c|} 
Topic \\
Sub-topic \\
Author \\
Age \\
Education \\
al level \\
Time \\
Space - \\
Geo \\
Learning \\
space
\end{tabular} & LMS logs & 400 & $\begin{array}{c}\text { Topical, } \\
\text { Personal and } \\
\text { Situational } \\
\text { Relevance }\end{array}$ & \begin{tabular}{|c|} 
Min-Max \\
Normalization \\
Technique \\
Z-score \\
Normalization \\
K-mean \& SOM \\
for clustering and \\
scatter plot
\end{tabular} & $\begin{array}{l}\text { TANAGRA } \\
\text { tool kit }\end{array}$ & \begin{tabular}{|c|} 
Cluster \\
cohesion \\
(SSE) \\
Cluster \\
Separation \\
(Squared \\
Error) \\
BSS (Between \\
cluster Sum of \\
Squares \\
TSS = WSS + \\
BSS \\
RS = \\
TSS/BSS \\
\end{tabular} & $\begin{array}{c}\text { RS }=0.73 \\
\text { to } 0.76 \\
\text { Significant } \\
\text { difference } \\
\text { between } \\
\text { clustered } \\
\text { groups }\end{array}$ \\
\hline $\begin{array}{c}{[20]} \\
\text { Descriptive } \\
\text { Study }\end{array}$ & 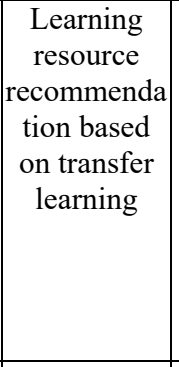 & \begin{tabular}{|l}
$\begin{array}{c}\text { Already } \\
\text { classified } \\
\text { new data }\end{array}$ \\
Old data
\end{tabular} & Web & - & $\begin{array}{c}\text { Users Interest, } \\
\text { Nearest } \\
\text { Neighbours } \\
\text { Top - N : } \\
\text { recommended } \\
\text { set } \\
\end{array}$ & \begin{tabular}{|c|} 
Statistical \\
Modelling: \\
Cosine Similarity, \\
Pearson \\
Correlation \\
Coefficient Metric \\
for related \\
similarity \\
Machine Learning \\
Algorithm \\
\end{tabular} & $\begin{array}{l}\text { Statistical } \\
\text { tools }\end{array}$ & $\begin{array}{c}\text { Users interest } \\
\text { in target } \\
\text { resources } \\
\text { feature set }\end{array}$ & \begin{tabular}{|l} 
Provides \\
Solution to \\
sparse \\
solution \\
collaborati \\
ve - \\
filtering \\
and cold \\
start-up \\
problem \\
\end{tabular} \\
\hline $\begin{array}{c}\text { [11] } \\
\text { Experiment } \\
\text { al Study }\end{array}$ & \begin{tabular}{|c|} 
Attribute - \\
based \\
recommende \\
r system for \\
Learning \\
Resource by \\
Learner \\
Preference \\
tree \\
\end{tabular} & $\begin{array}{c}\text { Historical } \\
\text { accessed } \\
\text { recourses } \\
\end{array}$ & $\begin{array}{c}\text { Metadata for } \\
\text { Architectural } \\
\text { Contents in } \\
\text { Europe }\end{array}$ & $\begin{array}{c}1148 \\
\text { Learners } \\
12000 \\
\text { resources }\end{array}$ & $\begin{array}{c}\text { Number of } \\
\text { neighbours (5- } \\
40)\end{array}$ & \begin{tabular}{|c|} 
MAE, MACE, \\
Normalized mean \\
absolute error, \\
Rank accuracy \\
metrics, Bayesian \\
Network, \\
Correlation \\
Learner \\
Preference Tree \\
\end{tabular} & $\begin{array}{l}\text { Statistical } \\
\text { techniques }\end{array}$ & \begin{tabular}{|c|} 
Precision, \\
Recall for \\
recommender \\
system \\
MAE for \\
prediction \\
quality metric
\end{tabular} & $\begin{array}{l}\text { Prediction } \\
\text { accuracy } \\
\text { improves } \\
\text { with } \\
\text { decreasing } \\
\text { sparsity }\end{array}$ \\
\hline $\begin{array}{c}{[21]} \\
\text { Suggestive } \\
\text { Framework } \\
\quad \text { for } \\
\text { recommend } \\
\text { er agent }\end{array}$ & \begin{tabular}{|c|} 
Track \\
learning \\
pattern and \\
personalise \\
using \\
adaptive \\
recommende \\
r agent \\
(IPBARA)
\end{tabular} & \begin{tabular}{|c|} 
Log of \\
navigatio \\
$\mathrm{n}$ \\
sequence \\
of learner \\
\end{tabular} & $\begin{array}{c}\text { LCMS } \\
\text { application } \\
\text { server }\end{array}$ & - & $\begin{array}{l}\text { Signature } \\
\text { pattern of } \\
\text { learners }\end{array}$ & \begin{tabular}{|} 
Concept manager, \\
pattern \\
recogniser, \\
User behaviour \\
analysis, generate \\
user navigational \\
patterns in \\
application env. \\
Algorithm : \\
gen_signature \\
pattern, \\
Gen_Repetitive_S \\
eq
\end{tabular} & $\begin{array}{l}\text { Mathematical } \\
\text { model used }\end{array}$ & $\begin{array}{c}\text { Generation of } \\
\text { concept map } \\
\text { tree by } \\
\text { concept map } \\
\text { manager } \\
\text { Generation of } \\
\text { signature } \\
\text { pattern by } \\
\text { Recommender }\end{array}$ & \begin{tabular}{|l} 
Successful \\
\\
\end{tabular} \\
\hline
\end{tabular}


International Journal of Science and Research (IJSR)

ISSN (Online): 2319-7064

Index Copernicus Value (2015): 78.96 | Impact Factor (2015): 6.391

\begin{tabular}{|c|c|c|c|c|c|c|c|c|c|}
\hline $\begin{array}{c}{[2]} \\
\text { Experiment } \\
\text { al } \\
\text { evaluation }\end{array}$ & \begin{tabular}{|c|} 
Personalised \\
recommenda \\
tion based \\
on Semantic \\
Analysis
\end{tabular} & \begin{tabular}{|l|} 
User logs \\
\end{tabular} & $\begin{array}{l}\text { Easy Learner } \\
\text { Website for } \\
\text { mathematics }\end{array}$ & $\begin{array}{l}30 \text { users } \\
170 \\
\text { learning } \\
\text { contents } \\
1325 \\
\text { learning } \\
\text { logs }\end{array}$ & $\begin{array}{c}\text { Performance } \\
\text { of } \\
\text { Collaborative } \\
\text { Filtering, } \\
\text { Sequential } \\
\text { Pattern } \\
\text { Mining, } \\
\text { Semantic } \\
\text { Analysis } \\
\text { Algorithm }\end{array}$ & $\begin{array}{c}\text { Video } \\
\text { Structurised } \\
\text { Description } \\
\text { lexical parsing } \\
\text { Semantic } \\
\text { Mapping } \\
\text { Rule Auto } \\
\text { Updating } \\
\text { mathematical } \\
\text { model }\end{array}$ & $\begin{array}{c}\text { Java, JSP, } \\
\text { SQL, Eclipse, } \\
\text { Apache } \\
\text { Tomcat } 6.0\end{array}$ & $\begin{array}{c}\text { Mean } \\
\text { Absolute } \\
\text { Error for CF, } \\
\text { SPM and } \\
\text { SAA }\end{array}$ & $\begin{array}{c}\text { MAE CF, } \\
\text { MAE } \\
\text { SAA, } \\
\text { MAE GSP } \\
\text { decreased } \\
\text { using RAU }\end{array}$ \\
\hline $\begin{array}{c}{[26]} \\
\text { Comparativ } \\
\text { e Study }\end{array}$ & \begin{tabular}{|c|} 
Predicting \\
Academic \\
performance \\
using \\
learning \\
analytics in \\
VLE \\
\end{tabular} & \begin{tabular}{|c|} 
Student- \\
system \\
Interactio \\
$\mathrm{n}$ Logs, \\
report \\
logs for \\
each \\
classificat \\
ion
\end{tabular} & $\begin{array}{c}\text { MOODLE } \\
\text { logs } \\
\text { interactions } \\
\\
\text { Data from } \\
\text { informal } \\
\text { learning } \\
\text { processes } \\
\text { outside VLE }\end{array}$ & \begin{tabular}{|c|}
20 to 30 \\
students, 2 \\
to 3 \\
teachers \\
100 hours \\
10 units
\end{tabular} & $\begin{array}{l}\text { Number of } \\
\text { interactions } \\
\text { for each } \\
\text { course } \\
\text { Moderating } \\
\text { factors like } \\
\text { Agent } \\
\text { Frequency } \\
\text { Mode }\end{array}$ & $\begin{array}{c}\text { Multiple linear } \\
\text { regression } \\
\text { between student } \\
\text { interactions } \\
\text { Variance of } \\
\text { dependent } \\
\text { variable as linear } \\
\text { combination of } \\
\text { independent } \\
\text { variables Data } \\
\text { analysis of } \\
\text { backward } \\
\text { multiple } \\
\text { regression }\end{array}$ & $\begin{array}{l}\text { SPSS } 18 \\
\text { (PASW) }\end{array}$ & \begin{tabular}{|c|} 
Average \\
interaction per \\
course for \\
each \\
classification
\end{tabular} & \begin{tabular}{|c|} 
No relation \\
between \\
creating \\
class \\
interaction \\
and final \\
academic \\
performanc \\
$\mathrm{e}$
\end{tabular} \\
\hline $\begin{array}{c}{[1]} \\
\text { Case Study }\end{array}$ & \begin{tabular}{|c|} 
User \\
Behaviour \\
Mining for \\
personalisin \\
g in e- \\
Learning \\
system
\end{tabular} & \begin{tabular}{|c|} 
Learner \\
behaviour \\
, learning \\
progress, \\
learning \\
resources \\
used, test \\
taken, \\
homewor \\
k library \\
content \\
\end{tabular} & $\begin{array}{l}\text { History of } \\
\text { learner } \\
\text { behaviour } \\
\text { Client side } \\
\text { Web logs } \\
\text { Server logs }\end{array}$ & $\begin{array}{c}\text { Not } \\
\text { mentioned }\end{array}$ & $\begin{array}{c}\text { Personalised } \\
\text { recommendati } \\
\text { ons }\end{array}$ & $\begin{array}{c}\text { Web- Browser } \\
\text { Plugin technology } \\
\text { If- then- else } \\
\text { model used for } \\
\text { browser plugin }\end{array}$ & $\begin{array}{c}\text { XML file for } \\
\text { learner history } \\
\mathrm{VC}++\end{array}$ & $\begin{array}{c}\text { Behaviour } \\
\text { mining in } \\
\text { personalised } \\
\text { recommendati } \\
\text { on engine }\end{array}$ & $\begin{array}{c}\text { Support for } \\
\text { individual } \\
\text { learning }\end{array}$ \\
\hline $\begin{array}{c}\text { [3] } \\
\text { Prototype } \\
\text { Design and } \\
\text { Evaluation } \\
\text { with } \\
\text { experimenta } \\
\text { tion }\end{array}$ & \begin{tabular}{|c|} 
Interoperabl \\
e Intelligent \\
Tutoring \\
System \\
\end{tabular} & \begin{tabular}{|c|} 
Student \\
Maths \\
Activity \\
Data
\end{tabular} & $\begin{array}{c}\text { Student } \\
\text { Interaction } \\
\text { with the } \\
\text { course and } \\
\text { tutor } \\
\text { interfaced } \\
\text { with } \\
\text { MOODLE, } \\
\text { Odijoo, SRTE } \\
\text { \& SCORM } \\
\text { Cloud }\end{array}$ & $\begin{array}{c}\text { Not } \\
\text { mentioned }\end{array}$ & \begin{tabular}{|c|} 
Grading Skills \\
Students : \\
Skillometer \\
$(0 \%-100 \%)$
\end{tabular} & \begin{tabular}{|c|} 
Comparison of \\
functionality and \\
features of LMS \\
and GRAPPLE \\
Approach, T- \\
Maestro \\
Approach and \\
Prototype
\end{tabular} & $\begin{array}{l}\text { PROLOG or } \\
\text { LISP for inner } \\
\text { and outer } \\
\text { Loop } \\
\text { Dreamweaver } \\
\text { IDE for web } \\
\text { development } \\
\text { RELOAD } \\
\text { IDE for } \\
\text { SCORM- PIF }\end{array}$ & \begin{tabular}{|c|} 
Functionalitie \\
s : - Inner \\
Loops - Outer \\
Loops \\
Features : \\
- Supports \\
- Provides \\
\end{tabular} & $\begin{array}{c}\text { Prototype } \\
\text { satisfied all } \\
\text { functionalit } \\
\text { ies and } \\
\text { features }\end{array}$ \\
\hline $\begin{array}{c}\text { [22] } \\
\text { Experiment } \\
\text { al study }\end{array}$ & \begin{tabular}{|c|} 
Student \\
classificatio \\
$\mathrm{n}$ for \\
academic \\
performance \\
using Neuro \\
Fuzzy Logic
\end{tabular} & \begin{tabular}{|c|} 
Four \\
categories \\
of data \\
good, \\
satisfacto \\
ry, good, \\
very good
\end{tabular} & $\begin{array}{l}\text { Questionnaire, } \\
\text { Quizzes on } \\
\text { Entrepreneurs } \\
\text { hip class in } \\
\text { JTETI UGM }\end{array}$ & $\begin{array}{c}71 \\
\text { responden } \\
\text { ts } \\
13 \\
\text { questions }\end{array}$ & $\begin{array}{l}\text { Percentage } \\
\text { value for } \\
\text { Categories }\end{array}$ & \begin{tabular}{|c|} 
Student \\
Classification \\
Model evaluated \\
using RMSE \\
Training data \\
processed by \\
ANFIS Editor \\
generating \\
Sugeno fuzzy \\
type and split the \\
membership \\
function \\
\end{tabular} & $\begin{array}{c}\text { ANFIS editor } \\
\text { on Matlab }{ }^{c e} \text { s } \\
\text { Fuzzy Logic } \\
\text { Toolbox }\end{array}$ & RMSE value & $\begin{array}{c}\text { Average } \\
\text { RMSE } \\
\text { after } 3 \\
\text { iteration : } \\
0.25611\end{array}$ \\
\hline $\begin{array}{c}{[9]} \\
\text { Descriptive } \\
\text { Analysis } \\
\end{array}$ & \begin{tabular}{|c|} 
Prototype \\
for \\
personalised \\
Recommend \\
ation based \\
on Hashtags \\
on e- \\
Learning \\
System \\
\end{tabular} & \begin{tabular}{|c|} 
User \\
behaviour \\
, user \\
profiles, \\
Datasets \\
in \\
Floksinor \\
y
\end{tabular} & $\begin{array}{l}\text { Web logs, } \\
\text { LMS logs }\end{array}$ & Not given & \begin{tabular}{|c|} 
Hash tag \\
definitions, \\
semantic \\
distance \\
between \\
definitions for \\
each hashtag
\end{tabular} & \begin{tabular}{|} 
Clustering groups \\
of similar \\
definition using \\
Markov \\
Clustering \\
Algorithm
\end{tabular} & $\begin{array}{l}\text { PDF to } \\
\text { organise } \\
\text { hashtags in } \\
\text { alphabetic } \\
\text { order }\end{array}$ & $\begin{array}{l}\text { Definition } \\
\text { sense } \\
\text { clustering }\end{array}$ & $\begin{array}{l}\text { Floksinory } \\
\text { Approachi } \\
\text { ng } \\
89.21831 \\
\text { with } \\
\text { ground } \\
\text { truth }\end{array}$ \\
\hline
\end{tabular}




\section{International Journal of Science and Research (IJSR) ISSN (Online): 2319-7064}

Index Copernicus Value (2015): 78.96 | Impact Factor (2015): 6.391

\begin{tabular}{|c|c|c|c|c|c|c|c|c|c|}
\hline $\begin{array}{c}{[8]} \\
\text { Experiment } \\
\text { al Case } \\
\text { study }\end{array}$ & \begin{tabular}{|c|} 
Design of \\
longest \\
common \\
subsequence \\
based on \\
genetic \\
algorithm
\end{tabular} & \begin{tabular}{|} 
Chapters \\
Groups of \\
LO \\
Results of \\
questionn \\
aire
\end{tabular} & $\begin{array}{c}\text { Courses } \\
\text { Groups based } \\
\text { on initial } \\
\text { sequence } \\
\text { Questionnaire: } \\
\text { bio-computing } \\
\text { students }\end{array}$ & $\begin{array}{c}7 \text { courses } \\
6 \text { groups } \\
4 \text { different } \\
\text { cases } \\
36 \text { results }\end{array}$ & $\begin{array}{l}\text { Common } \\
\text { sequences }\end{array}$ & \begin{tabular}{|c|} 
Algorithm \\
personalising Los \\
based on students \\
suggestions \\
Recommending \\
sequence using \\
fitness function, \\
mutation and \\
crossover \\
\end{tabular} & $\begin{array}{l}\text { Sequences } \\
\text { Proposed by } \\
\text { students }\end{array}$ & $\begin{array}{l}\text { Mathematical } \\
\text { model used }\end{array}$ & $\begin{array}{c}\text { High } \\
\text { efficiency }\end{array}$ \\
\hline $\begin{array}{c}{[16]} \\
\text { Experiment } \\
\text { al analysis }\end{array}$ & \begin{tabular}{c|} 
Dynamic \\
delivery of \\
learning \\
contents \\
using text \\
mining and \\
ontology \\
approach
\end{tabular} & \begin{tabular}{|} 
Learning \\
contents, \\
learning \\
log \\
activities, \\
forum \\
logs, quiz \\
scores
\end{tabular} & $\begin{array}{l}\text { Learning logs } \\
\text { from LMS }\end{array}$ & \begin{tabular}{|c|}
125 \\
learners of \\
scientific \\
writing \\
course \\
during 3 \\
weeks \\
Total \\
activity \\
logs: 7883 \\
\end{tabular} & $\begin{array}{l}\text { Quiz scores } \\
\text { Learning } \\
\text { material } \\
\text { preference for } \\
4 \text { groups of } \\
\text { students } \\
\end{array}$ & \begin{tabular}{|} 
Text mining using \\
deterministic \\
filtering rule, \\
clustering \\
Ontology \\
approach for \\
mapping learning \\
contents
\end{tabular} & $\begin{array}{l}\text { SPARQL to } \\
\text { match } \\
\text { learning style } \\
\text { Comparison } \\
\text { using charts }\end{array}$ & $\begin{array}{l}\text { Score: fair, } \\
\text { good, } \\
\text { excellent } \\
\text { Chart to } \\
\text { compare } \\
\text { learning } \\
\text { preference }\end{array}$ & $\begin{array}{c}\text { Higher } \\
\text { activity } \\
\text { participatio } \\
\mathrm{n}: \\
\text { excellent } \\
\text { scores }\end{array}$ \\
\hline $\begin{array}{c}{[25]} \\
\text { Framework } \\
\text { design and } \\
\text { experimenta } \\
1 \text { evaluation }\end{array}$ & \begin{tabular}{|c|} 
Framework \\
based on \\
fuzzy \\
learner \\
model and \\
optimised \\
Fuzzy Item \\
Response \\
Theory
\end{tabular} & $\begin{array}{c}\text { Learners } \\
\text { style } \\
\text { characteri } \\
\text { stics }\end{array}$ & $\begin{array}{l}\text { LMS data log, } \\
\text { Learning style } \\
\text { Questionnaire }\end{array}$ & $\begin{array}{l}40 \text { valid } \\
\text { learners }\end{array}$ & $\begin{array}{l}\text { Learners } \\
\text { satisfaction } \\
\text { feedback } \\
\text { Educational } \\
\text { sucess }\end{array}$ & $\begin{array}{c}200 \text { rules } \\
\text { generated for } \\
\text { courseware } \\
\text { recommendation } \\
\text { Learners ability } \\
\text { estimation } \\
\text { :Maximum } \\
\text { likelihood and } \\
\text { Bayesian } \\
\text { estimation } \\
\text { procedure used } \\
\text { for generating } \\
\text { item information } \\
\text { function } \\
\end{array}$ & $\begin{array}{c}\text { Mathematical } \\
\text { model } \\
\text { developed }\end{array}$ & \begin{tabular}{|} 
Questionnaire \\
: five point \\
Likert \\
Learners \\
ability : \\
Medium, \\
High
\end{tabular} & $\begin{array}{l}\text { More than } \\
83 \% \\
\text { learners } \\
\text { satisfied } \\
\text { and } \\
\text { showed } \\
\text { educational } \\
\text { progress }\end{array}$ \\
\hline $\begin{array}{c}{[18]} \\
\text { Experiment } \\
\text { al }\end{array}$ & $\begin{array}{l}\text { Learning } \\
\text { Style } \\
\text { prediction }\end{array}$ & \begin{tabular}{|c|} 
Normaliz \\
ed \\
Learning \\
style data, \\
learning \\
informati \\
on
\end{tabular} & $\begin{array}{l}\text { Log files } \\
\text { of learners }\end{array}$ & 50 & $\begin{array}{c}\text { Learning style } \\
\text { dimensions : } \\
\text { - Sequential/G } \\
\text { lobal } \\
\text { - Active/ } \\
\text { reflective } \\
\text { - Sensing/ } \\
\text { Intuitive } \\
\text { - Visual/ } \\
\text { Verbal } \\
\end{array}$ & \begin{tabular}{|c|} 
Classification \\
Clustering \\
learners \\
6 runs of Learning \\
Pattern \\
Recognition \\
\end{tabular} & $\begin{array}{c}\text { Cluster core } \\
\text { construction } \\
\text { algorithm } \\
\text { Simulated } \\
\text { Annealing } \\
\text { Algorithm }\end{array}$ & $\begin{array}{l}\text { Prediction } \\
\text { accuracy }\end{array}$ & $\begin{array}{c}90 \% \\
\text { accuracy }\end{array}$ \\
\hline $\begin{array}{c}{[12]} \\
\text { Experiment } \\
\text { al study } \\
\end{array}$ & \begin{tabular}{|c|} 
Personalised \\
Learning \\
Recommend \\
er System \\
using \\
Augmented \\
Reality \\
(AR) \\
browser for \\
fieldwork
\end{tabular} & \begin{tabular}{|} 
Preferenc \\
e \\
Thesauru \\
s each \\
consisting \\
of 165 \\
3DCG \\
browsing \\
behaviour
\end{tabular} & $\begin{array}{c}\text { Animals and } \\
\text { Plants 3DCG } \\
\text { database, } \\
\text { textbook } \\
\text { database, } \\
\text { Geometry } \\
\text { database for } \\
\text { Banff } \\
\text { National Park }\end{array}$ & $\begin{array}{c}1653 \mathrm{DCG} \\
\text { records } \\
70 \text { self } \\
\text { produced } \\
3 \mathrm{DCG} \text { of } \\
\text { animals } \\
\text { and plants }\end{array}$ & $\begin{array}{c}\text { Frequency of } \\
\text { 3DCG } \\
\text { manipulations } \\
: \\
\text { Transfer } \\
\text { Rotation } \\
\text { Scaling } \\
\text { Screenshot } \\
\text { Annotation } \\
\text { touch }\end{array}$ & \begin{tabular}{|c|} 
Mapping animal \\
and plant data to \\
geographical \\
information \\
Create term \\
behaviour matrix \\
Summing \\
behaviour vectors \\
normalised with \\
1-norm \\
Personal ranking \\
based on \\
similarity score \\
\end{tabular} & $\begin{array}{l}\text { Query in excel } \\
\text { Charts in } \\
\text { excel using all } \\
\text { results of } \\
\text { classification } \\
\text { of } \\
\text { personalised } \\
\text { ranking }\end{array}$ & $\begin{array}{c}\text { Observation } \\
\text { points : start } \\
\text { and end for } \\
\text { four users }\end{array}$ & $\begin{array}{c}\text { AR } \\
\text { browser } \\
\text { boost } \\
\text { motivation } \\
\text { in } \\
\text { fieldwork }\end{array}$ \\
\hline $\begin{array}{c}{[13]} \\
\text { Model } \\
\text { Proposal }\end{array}$ & \begin{tabular}{|c|} 
Recommend \\
er System \\
for assessing \\
student ${ }^{\text {es }}$ \\
activity for \\
supporting \\
e-Learning
\end{tabular} & \begin{tabular}{|} 
Students \\
activity \\
data via \\
API or \\
RSS
\end{tabular} & $\begin{array}{c}\text { Students of } \\
\text { University of } \\
\text { Rijeka, } \\
\text { Croatia } \\
\text { Web } 2.0 \text { tools }\end{array}$ & $\begin{array}{c}\text { Not } \\
\text { mentioned }\end{array}$ & $\begin{array}{c}\text { Impact of } \\
\text { recommender } \\
\text { on students } \\
\text { performance } \\
\text { during e- } \\
\text { tivities. }\end{array}$ & $\begin{array}{l}\text { Algorithms and } \\
\text { Rules for } \\
\text { generating } \\
\text { recommendation } \\
\text { on the basis of } \\
\text { activity, student, } \\
\text { group models } \\
\text { Surveys, } \\
\text { interviews } \\
\text { (students } \\
\text { satisfaction) } \\
\end{array}$ & $\begin{array}{c}\text { Web } 2.0 \text { tools, } \\
\text { SPSS }\end{array}$ & $\begin{array}{l}\text { Points per e- } \\
\text { cities for } \\
\text { control and } \\
\text { experimental } \\
\text { group }\end{array}$ & $\begin{array}{c}\text { System not } \\
\text { tested with } \\
\text { experiment } \\
\text { al group }\end{array}$ \\
\hline
\end{tabular}


International Journal of Science and Research (IJSR)

ISSN (Online): 2319-7064

Index Copernicus Value (2015): 78.96 | Impact Factor (2015): 6.391

\begin{tabular}{|c|c|c|c|c|c|c|c|c|c|}
\hline $\begin{array}{c}\quad[10] \\
\text { Recommen } \\
\text { der System } \\
- \\
\text { Experiment } \\
\text { ation } \\
\text { Case study } \\
\text { for } \\
\text { evaluation }\end{array}$ & $\begin{array}{c}\text { Fuzzy tree } \\
\text { matching } \\
\text { based } \\
\text { personalised } \\
\text { e-learning } \\
\text { Recommend } \\
\text { er System }\end{array}$ & $\begin{array}{c}\text { Movies } \\
\text { treated as } \\
\text { learning } \\
\text { activities } \\
\text { and } \\
\text { movie } \\
\text { users as } \\
\text { learners } \\
\text { Case } \\
\text { study: } \\
\text { learners } \\
\text { profile, } \\
\text { feedback, } \\
\text { subjects } \\
\text { data }\end{array}$ & $\begin{array}{l}\text { MovieLens } \\
\text { Data set } \\
\text { Case study: } \\
\text { random data } \\
\text { entered for } \\
\text { learners }\end{array}$ & $\begin{array}{c}2113 \text { users } \\
\text { rated } 20 \\
\text { movies } \\
\text { each } \\
\text { Case } \\
\text { study: } \\
5 \text { learners } \\
8 \text { subjects }\end{array}$ & $\begin{array}{c}\text { CF similarity } \\
\text { Ratings of } \\
\text { matched } \\
\text { learning } \\
\text { activity }\end{array}$ & $\begin{array}{c}\text { Fuzzy tree- } \\
\text { structured data } \\
\text { model to model } \\
\text { learners activities } \\
\text { and learners } \\
\text { profile } \\
\text { Seven step } \\
\text { recommendation } \\
\text { process }\end{array}$ & $\begin{array}{l}\text { Fuzzy } \\
\text { category tree } \\
\text { built for } \\
\text { sequential } \\
\text { relation } \\
\text { between } \\
\text { learning } \\
\text { activities } \\
\text { Case study: } \\
\text { Netbeans } \\
\text { development } \\
\text { platform, } \\
\text { JSF,EJB and } \\
\text { JPA } \\
\text { frameworks } \\
\text { PostgreSQL } \\
\text { database }\end{array}$ & $\begin{array}{c}\text { Mean } \\
\text { Absolute } \\
\text { Error assessed } \\
\text { and compared } \\
\text { with } \\
\text { Bobadilla }{ }^{\text {ees }} \\
\text { approach }\end{array}$ & $\begin{array}{c}\text { Accuracy } \\
\text { improved } \\
\text { by } 25.9 \%, \\
23.9 \% \text { and } \\
21.3 \% \text { on } \\
50 \%, 40 \% \\
\text { and } 20 \% \\
\text { testing sets }\end{array}$ \\
\hline
\end{tabular}

\subsection{Gaps in the Published Research}

After completing a review of more than hundredpapers in the field of ,Design of Intelligent tutoring systems using Enhanced Personalization in E-learning environmente, certain issues were found to be having a significant role in effective personalization for online learners. Some gaps found in the published research are:

- Learner"s information should be updated as the learner progresses through his e-learning course based on performances and behavior.

- Learner"s information could be collected and analyzed dynamically.

- Most of the researchers have proposed various recommender models but very few have provided experimental proof.

- It is necessary to keep track of learner"s performance and changes in the learning style and behavior and update his profile accordingly for effective personalization.

- It is desirable to build generic models that can be integrated to various Learning Management Systems for selecting and recommending appropriate learning objects to the learning.

\subsection{Strengths in the Published Research}

- Researchers worked in the area Personalization of elearning contents by collection information about the users using various e-learning platforms and mediums and then statically analyzing them using various techniques like Data Mining, Link analysis, Network analysis and also Statistical analysis like ANOVA for aligning the appropriate contents to the e-learners to meet their specific needs.

- Most of the Researchers used Data Mining techniques like clustering, classification, associations, prediction, ontologies and artificial neural network as solution approach for mapping learning objects to learners.

- Most Researchers have implemented the model developed by them using MOODLE and fetched good results.

- Some Researchers have also tried to dynamically map the contents to matching the learner"s profile, needs and capabilities.
- Researchers proposed to build recommender and intelligent tutoring systems considering learning styles of the learners.

\subsection{Limitations in the Published Research}

- Very few Researchers collected learner"s information on e-learning platforms dynamically.

- Very few researchers provide dynamic mapping of learning contents to the user.

- Most of the Researchers have used static methods of collection and analysis of user information.

- Most of the researchers proposed various models for recommendation and personalization but very few researchers have considered its implementation or provide experimental proof for the same.

\section{Discussion on Proposed Model}

The main objective of the proposed model

- To collect data related to Personalization parameters like user profile and Learning Styles and analyze them with reference to motivation and involvement for the learners from LMS.

- To create learning objects on the learning management system and group the learning objects into level.

- To select appropriate set of Personalization parameters and design a module to interface with LMS that includes feedback ensuing improved learning experience.

- To create ontology based mapping of learning objects based on students profile (static).

- To update the profile of the learner based on the behavior and performance of the learner.

- To implement and validate the model through some selected software and hardware setup.

\subsection{Methodologies/Technologies to be used}

The proposed model would be designed such that it can be integrated with any CMS or LMS, use the $\log$ files to classify the learners based on their capabilities using FelderSilverman"s learning style theory, using data mining techniques. Based on the learning style and capabilities of learner, learning objects would be displayed. The learner can then choose to take an assessment for the learning objects 


\section{International Journal of Science and Research (IJSR) \\ ISSN (Online): 2319-7064}

Index Copernicus Value (2015): 78.96 | Impact Factor (2015): 6.391

and based on the GPA score the learner can then choose to progress to the next level and choose the learning objects from the next level. This design is close to traditional teaching learning model as after completion of learning the student is allowed to take assessment to capture learning outcomes. It is self-paced as the learner chooses learning objects and then chooses the assessment pattern (could be subjective or objective) as desired by the learner. The next level of learning objects are displayed once the learner completes the current level like in the gaming scenario where the user is always motivated and engaged to take up new challenges.

\subsection{Proposed Software support}

The software proposed for model includes:

- Data Mining Techniques of Classification for classifying learners based on their capabilities.

- Middleware based on Mathematical Model applying fuzzy logic for mapping the learning objects to learners. Its main function being collection of data on learning behavior of the learners from the log files and update the same as and when the learner progresses through the course.

\subsection{Data Requirement}

- Historical data of learners collected using log files from LMS or CMS.

- Analysis of data to classify learners based on their capabilities

- Mapping learning objects to the specific learners applying fuzzy logic

- Evaluate learning outcomes of learners using the scores generated

- Capture and update progress of each learner

- Analyze the learneres capabilities to offer new level of learning objects to learners.

\subsection{Utilization of the outcome of Research}

This study will provide a design for a generic model that can be integrated with any Learning Management System so as to provide recommendation for displaying learning appropriate to the learning styles of the learners based on the previous learning outcomes captured in the log files. The model developed is close to the conventional teachinglearning model where learning outcomes are evaluated at each stage of learning when the learner progresses through his course. The study would be very useful to the instructor who desire to use a blended e-learning model and evaluate the learning outcome of individual student and at the same time allow self-regulated and self-paced e-learning which is a drawback with the conventional face-to-face model. The model is similar to the gaming model as it requires the learner to complete the previous level of training before selecting the next level learning objects therefore will motivate learners to take up new challenges during learning.

\section{Conclusion}

Review process was adopted in the area of e-Learning and different approaches of personalization using statically generated data collected on the e-Learning platforms and also dynamically generated data in the virtual environment of e-learning were reviewed with the aim of enhancing experience of e-learners. It was found that most of the researchers worked in the area personalization of e-learning contents by collection information about the users using various e-learning platforms and mediums and then statically analyzing them using various techniques like Data Mining, Link analysis, Network analysis and also Statistical analysis like ANOVA for aligning the appropriate contents to the e-learners to meet their specific needs. Some Researchers have used the above techniques for future prediction of the grades. Most Researchers have implemented the model developed by them using MOODLE and fetched good results. Some Researchers have also tried to dynamically map the contents to matching the learneres profile, needs and capabilities.From the above discussion, it is found that very few Researchers collected the learneres information on an e-learning platform dynamically. Very few researchers provide dynamic mapping of learning objects tousers. Most of the Researchers used static methods of collection and analysis of information. Comprehending all the above points it is found that more work can be done for analyzing the e-learner"s information and allocating the learning content dynamically. More work can be done to analyze the cognitive style of the learners and align the learning contents to their needs and requirements. Capabilities of Learning Management systems can be enhanced using tutoring or recommender systems for improved personalization of learning contents.

\section{References}

[1] Agudo-Peregrina, A.F.; Hernandez-Garcia, A.; Iglesias-Pradas, S., "Predicting academic performance with learning analytics in virtual learning environments: A comparative study of three interaction classifications," Computers in Education (SIIE), 2012 International Symposium on , vol., no., pp.1,6, 29-31 Oct. 2012

[2] Bhattacharya, S.; Chakraborty, A.; Basu, P.; Roy, S., "A framework for interactive pattern based adaptive recommender agent using concept map for personalized e-learning: IPBARA," Technology Enhanced Education (ICTEE), IEEE International Conference on , vol., no., pp.1,5, 3-5 Jan. 2012

[3] Bo Han; Xing-Wei Hao; Chao-Feng Liu, "The design and implementation of user behavior mining in Elearning system," Automatic Control and Artificial Intelligence (ACAI 2012), International Conference on , vol., no., pp.2078,2081, 3-5 March 2012

[4] Brown, E.J.; Brailsford, T.J.; Fisher, T.; Moore, A., "Evaluating Learning Style Personalization in Adaptive Systems: Quantitative Methods and Approaches," Learning Technologies, IEEE Transactions on , vol.2, no.1, pp.10,22, Jan.-March 2009

[5] Chellatamilan, T.; Ravichandran, M.; Suresh, R.M.; Kulanthaivel, G., "Effect of mining educational data to

\section{Volume 6 Issue 1, January 2017}




\section{International Journal of Science and Research (IJSR) \\ ISSN (Online): 2319-7064}

Index Copernicus Value (2015): 78.96 | Impact Factor (2015): 6.391

improve adaptation of learning in e-learning system," Sustainable Energy and Intelligent Systems (SEISCON 2011), International Conference on , vol., no., pp.922,927, 20-22 July 2011

[6] Cocea, M.; Weibelzahl, S., "Disengagement Detection in Online Learning: Validation Studies and Perspectives," Learning Technologies , IEEE Transactions on , vol.4, no.2, pp.114,124, April-June 2011

[7] Essaid El Bachari, El Hassan Abelwahed, Mohammed El Adnani, „An Adaptive Teaching Strategy Model in E-Learning using Learner"s Preference: LearnFit Framework ${ }^{e c}$, International Journal of Computer Science and Information Technology,(IJCSIT), vol. 3, pp.257-274, 2011

[8] Ghenname, M.; Abik, M.; Ajhoun, R.; Subercaze, J.; Gravier, C.; Laforest, F., "Personalized recommendation based hashtags on e-learning systems," ISKO-Maghreb, 2013 3rd International Symposium, vol., no., pp.1,8, 8-9 Nov. 2013

[9] Hidayah, I.; Permanasari, A.E.; Ratwastuti, N., "Student classification for academic performance prediction using neuro fuzzy in a conventional classroom," Information Technology and Electrical Engineering (ICITEE), 2013 International Conference on , vol., no., pp.221,225, 7-8 Oct. 2013

[10] HolenkoDlab, Martina, and NatasaHoic-Bozic. "Recommender system for web 2.0 supported elearning." In Global Engineering Education Conference (EDUCON), 2014 IEEE, pp. 953-956. IEEE, 2014

[11] Huayue Chen, "Personalized Learning Resources Recommendation Model Based on Transfer Learning," Computer Science and Electronics Engineering (ICCSEE), 2012 International Conference on , vol.2, no., pp.14,16, 23-25 March 2012

[12] Juan Yang; Zhi Xing Huang; Yue Xiang Gao; Hong Tao Liu, "Dynamic Learning Style Prediction Method Based on a Pattern Recognition Technique," Learning Technologies, IEEE Transactions on , vol.7, no.2, pp.165,177, April-June 2014

[13] Kasahara, M.; Takano, K.; Kin Fun Li, "A Personalized Learning System with an AR Augmented Reality Browser for Ecosystem Fieldwork," Advanced Information Networking and Applications (AINA), 2014 IEEE 28th International Conference on , vol., no., pp.89,97, 13-16 May 2014

[14] Lau, R.Y.K.; Dawei Song; Yuefeng Li; Cheung, T.C.H.; Jin-Xing Hao, "Toward a Fuzzy Domain Ontology Extraction Method for Adaptive eLearning," Knowledge and Data Engineering, IEEE Transactions on , vol.21, no.6, pp.800,813, June 2009

[15] LV Jia-Ke; Wang Xuan; Jiang Wei; Zou Xian-Chun; Wei Chao-Fu, "Design and Evaluation of a Multiagent System for Web Intelligent Tutoring," Computer Science and Software Engineering, 2008 International Conference on , vol.5, no., pp.885,888, 12-14 Dec. 2008

[16] Mahdi, B.J.M.; Fattaneh, T., "A Longest Common Subsequence based genetic algorithm for courseware design," E-Learning and E-Teaching (ICELET), 2013 Fourth International Conference on , vol., no., pp.40,46, 13-14 Feb. 2013
[17] Munoz-Organero, M.; Munoz-Merino, P.J.; Kloos, C.D., "Student Behavior and Interaction Patterns With an LMS as Motivation Predictors in E-Learning Settings," Education, IEEE Transactions on , vol.53, no.3, pp.463,470, Aug. 2010

[18] Nafiseh, S.; Ali, M.G., "Evaluation based on personalization using optimized FIRT and MAS framework in engineering education in e-learning environment," E-Learning and E-Teaching (ICELET), 2013 Fourth International Conference on , vol., no., pp.117,120, 13-14 Feb. 2013

[19] Nagori, R.; Aghila, G., "LDA based integrated document recommendation model for e-learning systems," Emerging Trends in Networks and Computer Communications (ETNCC), International Conference on , vol., no., pp.230,233, 22-24 April 2011

[20] Sabitha, A.S.; Mehrotra, D., "User Centric Retrieval of Learning Objects in LMS," Computer and Communication Technology (ICCCT), 2012 Third International Conference on, vol., no., pp.14,19, 23-25 Nov. 2012

[21] Salehi, M.; Kmalabadi, I.N., "Attribute-based recommender system for learning resource by learner preference tree," Computer and Knowledge Engineering (ICCKE), 2nd International eConference on , vol., no., pp.133,138, 18-19 Oct. 2012

[22] Santos, G.S.; Jorge, J., "Interoperable Intelligent Tutoring Systems as Open Educational Resources," Learning Technologies, IEEE Transactions on , vol.6, no.3, pp.271,282, July-Sept. 2013

[23] Shishehchi, S.; Banihashem, S.Y.; Zin, N.A.M.; Noah, S.A.M., "Review of personalized recommendation techniques for learners in e-learning systems," Semantic Technology and Information Retrieval (STAIR), 2011 International Conference on , vol., no., pp.277,281, 28-29 June 2011

[24] Sivakumar, P.; NandaKumar, G., "Personalization of Learning Objects for a course on Database Management System," Technology for Education (T4E), 2010 International Conference on , vol., no., pp.134,137, 1-3 July 2010

[25] Suryani, M.; Hasibuan, Z.A., "The study of dynamic delivery adaptive learning content in e-learning personalization using text mining and ontology approach ," Advanced Computer Science and Information Systems,2013 International Conference, vol., no., pp.21,26, 28-29 Sept. 2013

[26] Yi Li; Lin Mei; Jian Wang, "A personalized recommendation system in E-Learning environment based on semantic analysis," Information Science and Service Science and Data Mining (ISSDM), 2012 6th International Conference on New Trends in , vol., no., pp.802,807, 23-25 Oct. 2012

[27] Zaina, L.A.M.; Bressan, G.; Rodrigues, J.F.; Cardieri, M.A.C.A., "Learning Profile Identification Based on the Analysis of the User's Context of Interaction," Latin America Transactions, IEEE (Revista IEEE America Latina), vol.9, no.5, pp.845,850, Sept. 2011 